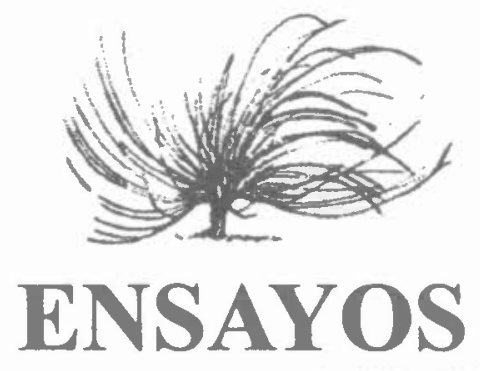




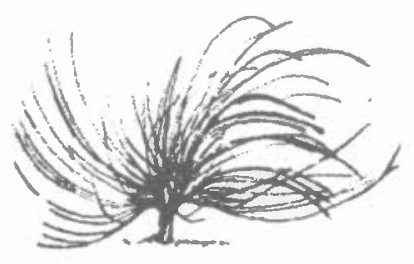

\title{
Reflexiones sobre pensamiento crítico en educación a partir de los planteamientos de Mc Laren, Giroux, Castells y Macedo
}

\author{
Luis Gerardo Meza Cascantel \\ Instituto Tecnológico de Costa Rica \\ Cartago, Costa Rica \\ gmeza@itcr.ac.cr
}

\section{Resumen}

Se presenta un análisis crítico, a partir de las lecturas de las obras de Giroux y de McLaren, orientado a esclarecer las prácticas que se han venido dando sobre la formación de educadores en las universidades, las cuales pretenden formar educadores constructivistas mediante procedimientos conductistas. El planteamiento de estos autores pueden ayudarnos a clarificar esta posición ideológica.

Palabras claves: Educación, formación de educadores, prácticas tradicionales, ideología dominante, proceso educativo, conocimientos crítico, política y poder.

\begin{abstract}
This article presents a critical analysis based on the Giroux and McLaren works. It is oriented to establish the different practices that have been used in the teachers' formation through behaviorist and constructivist approaches. This authors' analysis can help readers to clarify their ideological position.
\end{abstract}

Recibido: 08 de setiembre, 2010 - Aprobado: 29 de junio del 2011

1 Profesor y Doctor Catedrático en el ITCR. Es Director, docente e investigador en la Escuela de Matemática del Instituto Tecnológico de Costa Rica. Profesor II en la Universidad Nacional. 
Keywords: Education, teachers' training, traditional practices, dominant ideology, educational process, critical knowledge, politics and power.

\section{Mi punto de partida}

bordo este trabajo desde mi principal preocupación en este mo-
mento: la de formador de educadores. He contribuido a formar
profesores y profesoras de matemática por muchos años, tanto en la Universidad Nacional como en el Instituto Tecnológico de Costa Rica. La preocupación nace principalmente porque, unas veces mediante la investigación y otras mediante el contacto directo en los cursos de la licenciatura, he comprobado que la práctica de los educadores y de las educadoras que hemos formado no difiere, casi en nada, de las prácticas tradicionales. Desde luego, la preocupación va pareja con la convicción de que las prácticas tradicionales no son las más convenientes.

Por otra parte, desde hace mucho tiempo, incluyendo la etapa inicial de mi formación como educador, he escuchado que la Escuela es un aparato reproductor de la ideología dominante. En general he dado validez a este argumento, pero confieso que no había tenido completa claridad sobre cómo es que la Escuela cumple con ese papel. Me preguntaba cómo es que un profesor o una profesora transmite la ideología dominante, que es lo que él o ella hace que lo produce. Pensé en la forma en que damos lecciones, en las cosas que hacemos y decimos y no podía encontrar con claridad ese "cómo". Incluso me confundí más cuando conocí como se diseñaron los programas de matemática de la educación media, al comprobar que fueron formulados por funcionarios del MEP, algunos de los cuales fueron mis compañeros de formación, y que una parte del trabajo fue desarrollado por profesores y por profesoras de la Universidad de Costa Rica. La mayoría de estos colegas han trabajado conmigo en una cantidad importante de proyectos, son muy respetables en su desempeño profesional, y no parecen tener razones para privilegiar intereses de clases a las que no pertenecen.

¿Cómo, entonces, es que la Escuela sirve de aparato reproductor del sistema dominante? La lectura de las obras de Giroux y de McLaren, principalmente, me ha dado la pista. He comprendido que favorecemos el proceso reproductor porque actuamos convencidos de que hacemos las cosas que hay que hacer, sin cuestionar lo que hacemos y por qué lo 
hacemos. Tenemos una actitud reproductora porque transmitimos conocimientos elaborados por otros y en otras partes, como si fueran la única verdad posible. También porque miramos el proceso educativo como un proceso neutral, antiséptico, que no tiene vinculación con la política y el poder, en el cual creemos posible enseñar conocimientos sin incorporar elementos históricos, de género, de raza, etc. Actitudes como estas son, precisamente, las que necesita el sistema para perpetuarse. De paso, por la imposición de las normas y de los procedimientos instaurados en la Escuela, favorecemos la formación de personas sumisas, sin pensamiento propio, y en muchos casos, por el apego tan grande que generamos a las notas y a las calificaciones, convencidas de que lo que cuenta es "pasar", no importa cómo.

Por otra parte, las educadoras y los educadores no hemos tomado conciencia de los efectos que producen en las y los estudiantes, y en nosotros mismos, los medios de comunicación masiva: la TV, la radio, los periódicos, etc. y nunca, o casi nunca, consideramos que es tarea de la Escuela preparar a las y los jóvenes para estar en capacidad de responder críticamente ante lo que reciben de tales medios.

Como formador de educadores caigo en cuenta, además, de que nuestra propuesta también ha sido reproductora. Lo anterior porque nos hemos centrado principalmente en formar a las y los estudiantes en aspectos técnicos relacionados con los procesos de enseñanza- aprendizaje y hemos descuidado, casi absolutamente, el problematizar, el escuchar, el develar elementos ideológicos en los textos, en los programas de estudio, en las propuestas de reformas educativas, y hasta en develar nuestras propias prácticas cargadas de condicionantes ideológicos. Casi nunca partimos en nuestra labor formativa de las experiencias y de las necesidades de las y los estudiantes, ni analizamos los vínculos entre la Escuela y el poder político, ni historizamos el conocimiento, como propone Giroux.

Junto a la luz que me ha proporcionado estas lecturas para alcanzar una mejor comprensión del tema, debo agregar una aguda crítica, pienso que francamente merecida, que escuché hace pocos días de un estudiante, y que constituye un ejemplo de la falta de coherencia que a veces mostramos y que a la postre nos convierte en instrumentos de reproducción ideológica: los formadores de educadores en las universidades pretenden formar educadores constructivistas mediante procedimientos y prácticas conductistas. 


\section{Pensamiento crítico para McLaren, Giroux, Castells y Macedo}

Cuatro son los autores que me interesan, y es natural asumir que sus planteamientos no sean necesariamente idénticos en cuanto a lo que comprenden como pensamiento crítico. No obstante, me parece encontrar más semejanzas que diferencias, especialmente porque no he encontrado tema alguno en el que sostengan posiciones francamente antagónicas. Encuentro más bien diferencias de matices o en la profundidad con la que abordan cada problemática, y así lo asumo en este trabajo.

$\mathrm{Me}$ interesa resaltar algunas de las ideas particulares de cada uno de los autores y posteriormente presentar un análisis conjunto.

\section{Pensamiento crítico en Peter McLaren}

McLaren me ha parecido un escritor directo y erudito. Es directo porque escribe en una forma directa, esto es, no parece preocuparse por disimular o por dosificar sus planteamientos: va directamente al grano. Además, me ha parecido erudito por la forma profunda con la que trata los temas y por la cantidad impresionante de referencias y de citas que incluye en su obra. Me parece también que es un autor crítico que cree en la posibilidad de la transformación social.

Veo en McLaren una clara preocupación por aspectos sociales y culturales vinculados con el poder que se dan fuera del aula, así como por analizar la manera en que dichos elementos crean relaciones sociales dentro del aula. Lo esencial de su postura lo veo en su convicción de que la pedagogía crítica debe ser una estrategia y una respuesta poderosa a las condiciones históricas que nos han gestado como personas, y a las formas en que cotidianamente estamos insertos en las estructuras de poder existentes.

Pienso que McLaren muestra una actitud reflexiva que desnuda a la Escuela, no por un propósito morboso, sino por el contrario: con el fin de evidenciar el papel que juega como instrumento ideológico que permite transmitir, generación a generación, valores, creencias, apariencias y sensaciones, creando realidades que no son. Encuentro que su pensamiento crítico contribuye a mostrar a la Escuela como ese instrumento que somete a la población (estadounidense en su caso) haciéndole vivir una realidad que no es la verdadera, y señalando con valentía y con 
franqueza las tareas que la Escuela y que los educadores debemos asumir para revertir la situación. Me parece que un aspecto fundamental para McLaren está en la lucha que debemos librar por mejorar las condiciones de acción de las personas desposeídas y sometidas, y es su mérito plantear que en esta tarea la Escuela como institución y lo educadores como parte de ella, tenemos un papel (un nuevo papel) que cumplir.

Dentro de lo que estimo como elementos que configuran el pensamiento crítico de McLaren, está su señalamiento de que tenemos que rechazar las verdades recibidas, en su carácter de inmutables, ahistóricas, neutrales, antisépticas, lo mismo que ciertas convicciones aceptadas. En este punto creo que es profundamente importante su señalamiento, casi permanente, de que no debemos permitir que nos engañen quienes promueven una educación que elimina las diferencias, bajo el pretexto de eliminar las desigualdades. La concepción de buscar una Escuela que elimine las diferencias, asimilando el proceso a eliminar las desigualdades, es lo que permite las propuestas incontestadas de buscar la formación de las personas como si todos fueran hombres, blancos, etc., hecho que favorece el fracaso de muchas personas en el sistema educativo porque no lo son y no pueden serlo, y porque obvia las diferencias entre las personas y las diferencias asimétricas en la posesión del poder.

En el pensamiento crítico de McLaren encuentro también una profunda preocupación por mostrar que en el debate sobre la educación pública (estadounidense) se olvida la necesidad de examinar con detenimiento la forma como la Escuela contemporánea reproduce imágenes nacionales de ciudadanía inspiradas en la Ramborizacion de Norteamérica y plasmada en el Go for it (A por ello) de Rocky Balboa y en el Go ahead, Make my day (Adelante, dame el día) de Clint Eastwood, clichés que adornan la cultura brava de Estados Unidos. Esta cultura brava es una forma, pienso, de sometimiento de la población norteamericana que utilizan los gobernantes de ese país, y mediante ella legitiman la intervención armada en otras naciones como ha sucedido en América Latina, incluso en años recientes, o en otros continentes como en el caso de Irak.

Creo que en nuestro país también estamos olvidando debatir sobre este tipo de construcciones, que en nuestro caso, debe llevar doble propósito. Por un lado, analizar como la cultura brava estadounidense 
nos somete y nos afecta, legitimando en su propia población y en la nuestra, la intervención directa e indirecta de los Estados Unidos en nuestra propia acción. Tenemos que develar la propuesta sostenida de los gobiernos estadounidenses de que su cultura, su forma de gobierno, su forma de hacer las cosas, es la forma "que conviene" a los demás, cuando en el fondo sabemos que simplemente conviene a poderosos grupos comerciales. Por otro, tenemos que reflexionar sobre cuáles son los personajes con los que se identifican nuestros jóvenes, atomizados de "Rambos", Ricky Martin, héroes deportivos transitorios, etc. Tenemos que invitar en nuestras aulas a las y los estudiantes a analizar con qué personajes se identifican, por qué creen que lo hacen, y ayudarles a develar los elementos ideológicos que pueden estar presentes en tal identificación. Pienso como McLaren (1997) que es necesario empezar a historizar esta identificación en el contexto de los acontecimientos políticos y sociales que vive el país.

Relevante es el aporte de McLaren cuando reacciona contra lo que denomina "el pluralismo muerto", pues piensa que evita que se perciba la necesidad de historizar la diferencia, de reconocer la producción jerárquica de sistemas de diferencia y los intereses a los que dichas jerarquías sirven, y de percibir la diferencia como una construcción social forjada entre relaciones asimétricas de poder, de conflictos de intereses y en un clima de disentimiento y oposición.

Sobre este punto tenemos muchísimo que reflexionar y que actuar los educadores costarricenses. Bastantes mitos se han forjado sobre la amabilidad del pueblo costarricense con el extranjero y sobre la tolerancia a las ideas ajenas, por ejemplo. Lamentablemente en los últimos años, ante las fuertes inmigraciones de nicaragüenses, hemos visto que existe una fuerte cultura de rechazo a estas personas, por lo cual pienso que nos hemos mostrado como un pueblo poco receptivo de los extranjeros. La educación costarricense tiene pues el imperativo de formar a las y los jóvenes en este aspecto. Tarea también importante tiene la educación costarricense sobre la tolerancia a la opinión ajena, pero nos corresponde a las y los docentes profundizar sobre este tema y no manejarlo superficialmente en el ámbito de mito, dando por supuesto que es una característica adquirida del pueblo costarricense. Nos cabe aquí un pensamiento crítico que dude hasta de las verdades que hemos asumido como incuestionables. 
espacios en los que se dan complejos encuentros de culturas dominadas y subordinadas, cada un vinculada al poder que poseen para definir y legitimar una construcción particular de la realidad. Su pensamiento nos ilumina sobre que las Escuelas no son sitios neutrales en los cuales las y los estudiantes acceden a conocimientos neutros, despolitizados, sino que en ellas los diferentes valores, convenciones y conocimientos se acercan, mezclan, y excluyen en diferentes formas.

Este autor introduce elementos de reflexión sobre la política cultural, con la característica muy particular de elaborar un discurso pedagógico lleno de posibilidades.

Dentro de sus planteamientos considera que a los profesores se les enseña a utilizar numerosos modelos educativos o técnicas pedagógicas, pero no se les enseña como utilizar los instrumentos de análisis para reffexionar o elaborar críticas en torno a esos modelos y técnicas. Sostiene que en lugar de preocuparse por el dominio y el perfeccionamiento del uso de metodologías, los profesores y el personal de la administración deberían enfocar el problema de la educación examinando sus propias perspectivas acerca de la sociedad, las Escuelas y la emancipación.

Al reflexionar sobre los modelos de formación de educadores en nuestro país en los que he participado, llego a la conclusión de que la observación que Giroux plantea para el caso estadounidense se aplica también al muestro. Efectivamente, la formación de los educadores en Costa Rica enfatiza principalmente en el manejo de cuestiones pedagógicas (planeamiento didáctico, construcción de pruebas, elaboración de material didáctico, etc.) y no crea espacios para dotar a las futuras y a los futuros docentes de una capacidad de reflexión, de análisis o de crítica sobre lo que reciben, sobre lo que se les dice que deben hacer, etc.

A manera de ejemplo de los resultados que hemos obtenido con los modelos comentados anteriormente, podemos comprobar, lamentablemente, que una mayoría de los educadores graduados en estos procesos, una vez en el aula como profesionales, acuden a los libros de texto comerciales para apoyar su labor y los utilizan como si fueran una verdad incuestionable. He podido comprobar que los emplean sin cuestionar el enfoque didáctico, ni la precisión de los contenidos, ni la pertinencia pedagógica, etc. Lo mismo sucede, desafortunadamente, con el empleo de otros recursos didácticos como el software, las calculadoras o 
las computadoras. Desde luego, también nos cabe meditar sobre cuanto reflexionamos los propios formadores sobre los recursos que nosotros mismos utilizamos.

Para Giroux, el conocimiento no es objetivo, ni neutral, sino una construcción social que responde a unos intereses determinados. Con este aporte Giroux nos permite concebir que los diversos grupos sociales pueden participar en la transformación y construcción de una nueva realidad social.

Comparto con Giroux que más que tratar de evadirse de sus propias ideologías y valores, los educadores deberíamos evaluarnos críticamente con el fin de comprender de que manera nos ha conformado la sociedad como individuos, cuales son nuestras verdaderas creencias y como estructurar más positivamente los efectos que nuestra acción ejerce sobre los estudiantes y otras personas. Comparto su planteamiento, pero temo no estar muy claro sobre como hacerlo. En lo personal he tenido la oportunidad, a partir de estos estudios, de iniciar una toma de conciencia. No veo claro como puedo hacer para contribuir a que otros colegas puedan iniciar procesos similares, pero encuentro un mejor panorama en mi labor de formación de educadores.

Me parece relevante el análisis que hace Giroux de la educación como una forma de política cultural. Desde esta perspectiva, la enseñanza representa siempre unas formas de vida social, siempre está implicada en relaciones de poder y de prácticas sociales, y privilegia aquellas formas de conocimiento que proporcionan una visión especifica del pasado, del presente y del futuro. A partir de estos planteamientos concluyo que no podemos seguir enseñando como si tuviéramos la tarea de comunicar verdades inmaculadas, desprovistas de contenido social o histórico, y sin conciencia de que no podemos concebir la verdad, como dice Foucault, fuera del poder.

También considero relevante en Giroux su visión de que las Escuelas, entendidas como esferas públicas democráticas y los profesores como intelectuales transformativos, deben procurar, junto con los estudiantes, desarrollar un proyecto educativo que ahonde en el proceso democrático y en la eliminación de las desigualdades sociales, ya sea por razón de sexo, etnia, religión o clase social. 


\section{Pensamiento crítico en Manuel Castells}

Con frecuencia escuchamos sobre la necesidad del desarrollo científico y tecnológico para lograr el desarrollo nacional. Comparto que el desarrollo científico y el tecnológico son elementos que inciden en el desarrollo nacional, en general, y en el mejoramiento de las condiciones de vida del pueblo costarricense en particular.

Es motivo de preocupación, no obstante, que se asuma una posición radical sobre el hecho de que el desarrollo tecnológico, cada vez más vertiginoso, conducirá a la humanidad a mejores condiciones de vida. Castells muestra una posición clara que nos indica que no es necesariamente así. Señala con firmeza que la sociedad de la información nos ha conducido, proceso que se sigue incrementado, a vivir de manera acelerada, solitaria y con grandes desequilibrios. A la vez, identifica también elementos sociales que están alterando fuertemente el concepto de familia: el alto índice de divorcios y las posibilidades alternativas de procreación (fecundación in Vitro y las madres de alquiler). Además, insiste en los importantes procesos de transformación social ligados a diferentes movimientos sociales (feminista, gay-lésbico, ecologista, entre otros) que introducen nuevas formas de relaciones humanas.

Según su planteamiento la sociedad que se está generando no es una sociedad más justa, pues, en particular, se están disolviendo las instituciones sociales. Supone que las nuevas formas de relación social del futuro estarán construidas por estrategias individuales. Visto de esta manera el panorama, piensa que es sumamente necesario apelar a la ética, a la solidaridad y al humanismo, pues de esto dependerá el tipo de sociedad que se podrá configurar.

¿Tiene su visión alguna pertinencia con lo que vemos suceder en nuestro pais? En principio sabemos que Castells trabaja y reside en los Estados Unidos, aunque es español de nacimiento. Por eso podriamos pensar que sus planteamientos valen principalmente para los Estados Unidos o para los países desarrollados. En lo personal pienso que sus planteamientos cubren a todo el planeta, porque, tal y como el mismo señala, el fenómeno no es sólo para las potencias, sino que involucra a todos los países del mundo. Cabe esperar, no obstante, consecuencias en el corto plazo distintas para los diferentes países.

Los planteamientos de este autor son importantes porque analiza elementos que podríamos dejar de mirar en la vorágine del desarrollo 
tecnológico. Nos hemos involucrado en un vertiginoso proceso de desarrollo, Costa Rica no es excepción, en el que cada día hay novedades de tipo tecnológico, que nos afectan en nuestra forma de trabajar, de vivir en el hogar, de comunicarnos con las personas, de aprender, etc. El asunto es impresionante. En este mundo acelerado hemos ido perdiendo valores y tal vez lo más grave, no tenemos conciencia de cuantas cosas han cambiado o están cambiando, valores y principios incluidos, y no tenemos ideas claras de cómo nos va a afectar como personas o como grupos sociales estos procesos. Tampoco hemos dimensionado correctamente la manera en la que el aporte de diversos grupos sociales han reconfigurado las relaciones en la sociedad.

El pensamiento crítico de Castells sobre estos fenómenos es un punto de partida.

\section{Pensamiento crítico en Donaldo Macedo}

Macedo explicita en su trabajo como la selección de los contenidos no es un proceso neutro. En este caso particular lo hace demostrando como lo que se incluye en una obra particular (Alfabetización cultural: lo que todos los americanos necesitan saber, de E.D. Hirsch) intencionalmente selecciona como verdaderos unos hechos, y oculta otros.

El pensamiento crítico de Macedo, juzgo a partir del artículo leído, resalta varios elementos importantes. Por una parte, ya mencionado, los elementos intencionales presentes en la selección de los contenidos. Por otra, evidencia que el control ideológico en la escuela se lleva a cabo tanto por medio de la omisión de hechos, como por la presentación engañosa de los mismos.

Desde luego, comparto con este autor, que en la selección de los contenidos se omiten referencias a hechos históricos. A veces con el fin de resaltar algunos aspectos, otros con el fin de ocultar hechos que podrían llevar a ciertos tipos de reflexión que no conviene generar. Pienso que dentro de estas estrategias se incluye el presentar la información con ribetes exagerados o caricaturizados. Es el caso de mostrar la información en el ámbito de cuento, de historia que no tiene vínculos con procesos humanos en los cuales se sufrió, se lloró, se sintió, hubo alegrías, hubo éxitos o fracasos: elementos muy humanos.

Resalto en Macedo su aporte para configurar una educación que respete e integre las diferencias culturales. 
Análisis

En general, creo que para estos autores el pensamiento crítico está constituido por una actitud de permanente reflexión y cuestionamiento, en la cual no caben posiciones neutrales, sobre la cotidianeidad. Giroux, McLaren y Macedo muestran referencias más explícitas a la educación (incluyendo el trabajo en el aula), mientras que Castells trata más sobre el impacto de las tecnológicas de la comunicación y de la información, y sobre los manejos ideológicos que se esconden tras ellos. Todos, eso sí, se muestran preocupados por los efectos en el desarrollo social y en las injusticias que se dan en la época actual, que acentúan las desigualdades sociales y en la que persisten mecanismos (la educación es uno de ellos) que favorecen la existencia de una hegemonía que permite a ciertas clases sociales perpetuarse en sus privilegios.

Es de tener en cuenta que los planteamientos de los autores parten de su vivencia, trabajo y conocimiento de la sociedad estadounidense, aunque sólo Giroux sea nativo de los Estados Unidos. La sociedad estadounidense guarda enormes diferencias con la nuestra, y en consecuencia, cabe esperar que también su educación y sus propósitos. No obstante, pienso que es posible y necesario, entresacar de los planteamientos de estos autores algunos elementos a partir de los cuales pueda reflexionar sobre nuestra propia educación, y en particular, sobre mi propia práctica como educador y como formador de educadores.

De esta manera, he podido reflexionar sobre cómo es que la educación formal costarricense reproduce las estructuras de dominación. En efecto, he podido pensar que la existencia de ciertos ritos, costumbres, y procedimientos nos llevan, a las educadoras y a los educadores costarricenses, a caer en una rutina, a estar preocupados permanentemente por terminar los programas, por cumplir con listados de contenidos, por asegurar que nuestros estudiantes puedan presentar "exitosamente" las pruebas de sexto grado, noveno o de bachillerato, lo que nos consume en un "hacer" que, además de reproducir cierto tipo de prácticas y de construir cierto tipo de subjetividades (lo que es bueno, lo que es malo, lo que debe hacerse y lo que no, a lo que podemos aspirar y a lo que no, lo que debemos resaltar y lo que no), nos elimina casi por completo la posibilidad de reflexionar sobre lo que hacemos, como lo hacemos y por qué lo hacemos. 
Tampoco nos cuestionamos sobre los textos que usamos, sobre su abordaje de los contenidos, del tratamiento que se da al conocimiento (absoluto, invariante, construido por seres humanos sin contexto, ahistórico) ni reflexionamos sobre otros aspectos que se nos imponen: forma de evaluar, listados de contenidos, procedimientos de aula. La reflexión es poca aun sobre aspectos claramente contradictorios: constructivismo evaluado por pruebas de corte conductista.

Desde luego, dándole crédito a Giroux, a pesar de este procedimiento casi mecánico con el que actuamos en nuestras aulas siempre se dan elementos de resistencia, pero no existe conciencia de nuestro papel reproductor.

Me parece claro que estos cuatro autores comparten una preocupación, una actitud y una acción que refleja un interés por fortalecer a los débiles y por transformar las desigualdades y las injusticias sociales. $\mathrm{Y}$, algo muy importante, ubican a la Escuela y a los educadores como elementos sustantivos en la lucha por lograr esos propósitos. Este es un elemento que, de alguna manera, pienso que en lo personal había olvidado: nos corresponde como educadores asumir, no solo la preocupación, sino también la acción, para buscar como mejorar las condiciones de acción de las personas menos favorecidas. Esta acción conlleva, desde luego, el crear conciencia en nuestros estudiantes del para qué de la educación, de las interferencias ideológicas presentes en la Escuela, en la propaganda, etc. y en gestar un pensamiento reflexivo, que les permita dudar sistemáticamente de lo que ven, de lo que oyen, de lo que se les presenta como verdades innegables, para que busquen, a partir de su propia reflexión, el significado de las cosas.

Es claro que estos autores ven a la Escuela no sólo como un mecanismo de reproducción de las estructuras económicas y sociales capitalistas, sino que la conciben como un espacio de reacción y resistencia.

A partir de las lecturas realizadas, que estos autores conciben como pensamiento crítico, entiendo la necesidad de considerar la educación en un contexto histórico, examinando a las Escuelas, además, como parte de las relaciones sociales y políticas que caracterizan a la sociedad dominante. En este sentido, los autores indican claramente que no cabe mirar a las Escuelas como lugares neutrales en las que es posible dotar de conocimientos a los jóvenes, partiendo de una neutralidad que no existe: no hay neutralidad en la selección de los contenidos, ni de los textos, 
ni en la forma que se organiza la Escuela, ni en la selección de metas, ni en los patrones de conducta que se consideran aceptables, ni en otros muchos aspectos.

Por eso, me parece relevante asumir con los autores que la concepción tradicional de la enseñanza y aprendizaje como un proceso neutral o transparente, antisépticamente extraído de los conceptos de poder, política, historia y contexto, ya no puede ser asumido como tal. Por tanto, pienso que, pensamiento crítico es, para los autores, asumir que las Escuelas son algo más que simples lugares de instrucción.

Así, a partir de los planteamientos de los autores, cabe mirar a la Escuela como un lugar donde ideologías y formas sociales heterogéneas colisionan en una arrolladora lucha por la dominación. Pienso, siguiendo a Giroux, que las Escuelas no son sólo instrumentos de dominación, sino que siempre ofrecen elementos de resistencia. Potenciar este elemento de resistencia y convertirlo en un instrumento de transformación social es un imperativo que se extrae de los planteamientos de los autores (Giroux y McLaren, especialmente), con el cual me identifico.

\section{Reflexión}

Vivo en Costa Rica. Creo conocer bastante del sistema educativo costarricense porque me he desempeñado como profesor de matemática en la educación secundaria pública, como profesor universitario en dos universidades estatales y una privada, y porque, mediante el desarrollo de programas de capacitación y otras intervenciones académicas, mantengo contacto con profesores y profesoras de la educación media y sus estudiantes. Además, he realizado estudios formales en tres universidades estatales costarricenses.

A partir de estas vivencias percibo en los colegios una actitud rutinaria, una premura en las y los docentes por cubrir programas, por preparar a las y los estudiantes para el examen de noveno o de bachillerato, una escasez de materiales y de otros recursos. Noto también una oferta bastante clásica, repetitiva cada día y cada año. Observo que las lecciones que se imparten este año no difieren de las que he observado hace uno, dos, tres o tal vez veinte años. Como vemos en la prensa, el señor Ministro hace defensa insistente de algunos elementos que, tal vez ingenuamente, algunos identifican como elementos de calidad educativa (200 días lectivos al año, por ejemplo). Otros expresan con crudeza su 
desacuerdo con el "pedagogismo", al cual parecen identificar como el causante de los males de la educación nacional.

¿Cómo es que el sistema educativo convierte al o la docente, rápida y suavemente, en seres pasivos, indiferentes, buscadores de excelencia a veces pero con concepciones atrofiadas de lo que se busca? Por una parte, tal como expuse anteriormente, creo que el sistema educativo está cargado de ritos, de costumbres, de formas "correctas" de hacer las cosas, sobre las que no se reflexiona mucho. El o la docente se ve saturado de procedimientos que debe acatar y que le marcan el camino, y que con el tiempo, llegan a percibirse como "naturales". Por otra parte, el sistema educativo formal termina siendo visto como el único capaz de llenar las necesidades educativas de las personas, esto nos lleva a mirar como imposible una Escuela distinta a la que tenemos: los procedimientos, organización, etc. de la Escuela llegan a ser percibidos por los propios maestros como inevitables.

Confieso que los mismos formadores de educadores llegamos también a mirar como "marco definido" el sistema educativo costarricense, y es para ese mismo sistema, críticas casi ausentes, que formamos a las y los educadores: la vinculación con el sistema educativo, cuando se da, se da para reproducirlo y no para transformarlo.

Por lo anterior, creo que los docentes llegamos a percibir el sistema educativo como algo que es, que no puede ser diferente, y es precisamente esta forma de pensamiento la que necesita el sistema para seguir operando en su papel reproductor de la ideología dominante. No vemos como transformar el sistema, a lo que damos espacio y a lo que nos hemos acostumbrado, es a las "reformas educativas", consistentes en cambios cosméticos de nombres rimbombantes, que en el fondo no cambian nada pero que parecen prometer mucho. Se trata de "lavados de cara" que en el fondo no cambian nada. Un ejemplo de lo anterior, es la declaración del constructivismo como una de las fuentes filosóficas que sustentan la política educativa vigente. No obstante, al mirar la práctica del aula o al confrontar medidas adoptadas o mantenidas por el sistema, nos llevan a concluir que la práctica educativa no es de carácter constructivista: sigue siendo predominantemente conductista.

A partir de los planteamientos de los autores cuyo pensamiento es objeto de este trabajo, he tomado conciencia de otro hecho también muy importante: la suspicacia que hay que tener para no dejarse engañar por propuestas que en apariencia resuelven problemas sociales, pero que 
en el fondo sólo legitiman los mismos. Ya con los planteamientos de Flecha había tomado cuenta de ello, pero ahora, a partir de Giroux y de McLaren especialmente, me doy cuenta de que ciertas prácticas podrían confundirse con verdaderas alternativas de cambio.

Pienso que no sólo es necesario que los educadores tomen acción como intelectuales transformadores, asumiendo actitudes críticas y acciones consecuentes, sino que se requiere un estado de reflexión muy especial para develar los elementos ideológicos que nos muestran una realidad que no es.

También es relevante, en la posición de los autores comentados, desarrollar un pensamiento crítico que nos permita distinguir las interpretaciones que están ideológicamente distorsionadas de las que no lo están.

\section{Conclusiones}

A manera de conclusiones podemos indicar lo siguiente:

1. Uno de los elementos más impresionantes de McLaren (1997) es su convicción de que la enseñanza para el fortalecimiento personal y social es éticamente previa a cuestiones epistemológicas o al dominio de las habilidades técnicas o sociales que son priorizadas por la lógica del mercado.

Me ha impresionado porque, de pronto, siento descubrir que este no es un principio fundamental en la educación costarricense en la cual seguimos concentrados principalmente en los contenidos y en las destrezas. Me ha impresionado porque me he sentido señalado, en mi papel de educador y de formador de educadores, de no haber meditado y considerado un principio tan fundamental: primero la persona. Algunos elementos del sistema educativo costarricense, me hacen concluir que un principio como este no es significativo en nuestro medio: para muestra pensemos en la sacrosanta importancia que tiene el examen de bachillerato.

2. Los autores analizados, McLaren y Giroux en particular, rechazan la consideración general de que la Escuela constituye el principal espacio para la movilidad económica y social, argumentando en su lugar que la enseñanza norteamericana ha fallado en su promesa de llevar a cabo una reforma igualitaria. Desde esta 
perspectiva, los resultados económicos, sociales y políticos de la enseñanza son mucho mayores para los económicamente acomodados que para los desaventajados.

Me he puesto a pensar como es la situación en Costa Rica. La verdad es que siempre he creído que la educación costarricense ha sido, efectivamente, un vehículo de movilidad social gracias al cual muchas personas hemos podido ascender en la escala social y alcanzar posiciones, tanto sociales como laborales, superiores a las de nuestros padres. Precisamente esta es una de las características que sigo viendo en la escuela pública y que me hace defensor a ultranza de ia universidad estatal. Reconozco que en los últimos años la educación, por uno de los efectos perversos de la globalización, ha perdido importancia como elemento de movilidad social: cada vez es menos seguro ascender en la escala social al subir en la escala educativa. No tenge en este momento la información necesaria para poder valorar en forma más profunda si las preocupaciones expuestas por los autores también se dan en nuestro país, tengo la esperanza de que no sea así.

Me preocupa no obstante, que subsista en Costa Rica, al igual que en otros paises, dos concepciones que tienen profundos elementos ideológicos. En primer lugar, que quienes no participen del sistema educativo formal están prácticamente condenados a sufrir de ciertos elementos de injusticia social, como tener que asumir trabajos mal remunerados, con pocas garantías laborales. Por otra, la creencia de que quienes desertan del sistema educativo son culpables de su decisión, obviando elementos sociales y políticos, que influyen de manea poderosa en este tipo de decisiones. Miramos la deserción del sistema educativo como un problema individual y no como un problema social.

3. Tiene también importancia, desde mi punto de vista, el planteamiento de los autores de que los educadores críticos se niegan a aceptar la función que el capitalismo les asigna como intelectuales, profesores y teóricos sociales: servir a los postulados ideológicos e institucionales existentes en los centros públicos de enseñanza y simultáneamente, reducir los valores y habilidades de los grupos minoritarios. 
Tal como he expuesto en otras partes de este trabajo temo que la mayoría de las y los docentes costarricenses no hayamos adquirido plena conciencia del papel reproductor que asume la escuela, y del papel que jugamos en esa maquinaria. No se trata de un asunto de falta de mística, pues creo que la mayoría de las educadoras y educadores de nuestro país la tienen, y hacen un gran esfuerzo por hacer un buen trabajo. El asunto es otro. No tenemos plena conciencia de que al hacer las cosas que hacemos, al meditar poco o nada sobre lo que hacemos, al no cuestionar por qué lo hacemos o por qué tenemos que hacerlo, al no luchar por mayores posibilidades para decidir sobre aspectos sustantivos de nuestra propia labor, estamos contribuyendo a sostener un sistema de desigualdades, en el cual existen injusticias sociales.

4. La pedagogía crítica argumenta que la principal función de la Escuela es la de reproducir los principios, valores y privilegios de las élites existentes. Este punto es muy agudo, sin duda. Alguna vez escuché que, ante los resultados tan malos que arroja la escuela, pues las personas en general no parecen aprender ni contenidos ni habilidades básicas de lectura, comunicación oral u escrita, etc., sólo se explica que los gobiernos sigan invirtiendo cantidades millonarias en el mantenimiento de la escuela por el excelente papel que realiza como reproductora del sistema.

5. Es claro en el mensaje de los autores, particularmente en Giroux, McLaren y Macedo, que la pedagogía crítica se compromete con formas de aprendizaje y de acción que son adoptadas en solidaridad con los grupos subordinados y marginados.

Lo anterior significa que la pedagogía crítica busca el autofortalecimiento y la transformación social, cuestionando lo que se da por hecho, o lo aparentemente evidente o inevitable, en la relación entre las Escuelas y el orden social.

Este segundo párrafo me hizo pensar en los planteamientos de Carr y Kemmis, quienes plantean que el profesor crítico tiene que cuestionar aun lo que ha considerado siempre incuestionable.

He pensado también en cuantas cosas damos por "naturales" las y los docentes. Año con año celebramos, por ejemplo, las llamadas fechas patrias con el mismo tipo de actividades, con las 
mismas frases, con los mismos mensajes, y nunca, o casi nunca, nos cuestionamos el verdadero sentido de la acción que estamos celebrando, ni nos cuestionamos, incluso, si lo que hemos venido repitiendo por años se ajusta a los hechos históricos.

A manera de ejemplo, tenemos el caso de la celebración del 11 de abril, día en el cual festejamos a nuestro héroe nacional. Las niñas y los niños reciben un mensaje sobre el personaje que lo lleva casi en el ámbito de mito, pero la mayoría de las educadoras y de los educadores no tienen claro cual puede ser el origen de esta celebración: los cafetaleros de la época necesitaban un héroe extraido de las clases sociales más humildes, para legitimar de esta manera que sus propios hijos no fueran a la guerra.

6. McLaren y Giroux, especialmente, señalan que algunos de los autores críticos se han esforzados más por hacer señalamientos críticos al sistema, pero se han mostrado incapaces de dar orientaciones concretas sobre los cambios que deben introducirse. En este sentido he encontrado en estos cuatro autores una perspectiva distinta. En efecto, McLaren y Giroux hacen aportes concretos sobre las posibilidades de la escuela y de los educadores para asumir tareas de transformación social. McLaren, por ejemplo, señala la necesidad y la importancia de que en la escuela se permita a los estudiantes analizar sus identificaciones con los super-héroes de la ficción (Rambo, por ejemplo), para que puedan explicitar porque se identifican con ellos, o sugiere como tarea curricular importante preparar a las y los estudiantes para que puedan responder críticamente a lo que reciben de los medios de comunicación masiva.

Giroux hace también aportes concretos, algunos de los cuales comentamos de manera más profunda en este trabajo. Creo que su visión del educador como intelectual transformador, su propuesta de las escuelas como esferas públicas democráticas o sus señalamientos sobre algunas de las características que deben poseer los planes de formación de educadores, son algunas de ellas.

En Macedo y en Castells también encontré elementos concretos. El primero, desnuda los elementos ideológicos presentes en la selección curricular, por ejemplo. El segundo, apunta valiosos elementos, al señalar la necesidad de crear nuevos mecanismos 
de relación social que permitan incorporar las nuevas características de una sociedad de la información, en la cual se han gestado nuevas formas de relaciones sociales producidas por el nuevo papel que juega la mujer en el proceso productivo, la existencia de elementos que afectan seriamente el concepto tradicional de familia (como los divorcios o las nuevas formas de procreación) y el aporte de grupos organizados que aportan nuevas formas de relación personal como los movimientos feministas, los ecologistas o los grupos gay-lésbicos.

7. La idea de profesorado como intelectualidad transformadora sugiere que los educadores y educadoras resalten y hagan problemáticos los intereses políticos e ideológicos que estructuran sus trabajos. Los educadores y las educadoras deben reconocer que, ni el conocimiento que enseñan ni los métodos que seleccionan para enseñar, son neutrales o carentes de significados ideológicos. Es importante que las profesoras y los profesores reflexionen permanentemente sobre lo que enseñan, como lo enseñan y por qué lo enseñan.

En otras palabras, las profesoras y los profesores tenemos la responsabilidad de evitar que la misma cotidianeidad nos impida tomar conciencia y comprensión de nuestra propia alienación.

\section{REFERENCIAS}

Castells, Manuel, Flecha, Ramón, Freire, Paulo, Giroux, Henry, Macedo, Donaldo y Willis, Paul (1994). Nuevas perspectivas críticas en educación. Barcelona: Ediciones Piados Ibérica, S.A.

Giroux, Henry (1998). La escuela y la lucha por la ciudadania. Pedagogia crítica de la época moderna. México: Siglo Veintiuno Editores S.A.

Gutiérrez, Francisco ( 1981). Strip Tease de la escuela. Heredia: EDITORIALPEC.

McLaren, Peter ( 1997). Pedagogía critica y cultura depredadora. Políticas de oposición en la era posmoderna. Barcelona: Paidós.

McLaren, Peter (1998: 91-294)- Pedagogía critica: un panorama general. En: La vida en las escuelas, una introducción a la pedagogía crítica en los fundamentos de la educación. México: Siglo Veintiuno Editores S.A. 